\title{
Atomic-scale modeling of next-layer nucleation and step flow at the Ge(105) rebonded-step surface
}

\author{
S. Cereda and F. Montalenti* \\ L-NESS, Dipartimento di Scienza dei Materiali della Università degli Studi di Milano-Bicocca, Via Cozzi 53, I-20125 Milano, Italy
}

(Received 26 January 2007; published 17 May 2007)

\begin{abstract}
Based on ab initio calculations, we propose an atomic-scale path leading to the growth of a new layer on the rebonded-step reconstructed Ge(105) surface. We show that the nucleation of (001)-like dimers triggers the formation of low-energy adtrimers within the surface unit cell. The presence of adjacent trimers and the arrival of a further adatom initiate a fast kinetic process, allowing the perfect rebonded-step structure to be rebuilt. After repeating some of the calculations under compressive-strain conditions, we discuss position-dependent nucleation in (105) Ge pyramid on $\mathrm{Si}(001)$, finding solid theoretical justification for the experimentally observed preferential nucleation at the top of Ge islands, followed by fast step flow.
\end{abstract}

DOI: 10.1103/PhysRevB.75.195321 PACS number(s): 68.55.Ac, 68.47.Fg, 68.43.Bc, 68.35.Fx

\section{INTRODUCTION}

A detailed theoretical understanding of the elementary microscopic processes occurring during growth is required in order to build accurate and reliable models, ultimately helping in determining the typical experimental conditions under which the desired result, in terms of film morphology and/or chemistry, can be obtained. ${ }^{1}$ Even in the presence of simple geometries and nondirectional bonds, highly nontrivial kinetic processes can occur, as it was demonstrated by several experimental and theoretical results on fcc (001) metal surfaces (see, e.g., Refs. 1-4). This evidence calls for a careful and accurate analysis of possible atomic-scale mechanisms.

Due to the high energetic cost related to dangling-bond formation, semiconductor surfaces tend to display major reconstructions, ${ }^{5}$ where the atomic arrangement in the outermost layers strongly differs from the bulk crystal one. As a consequence, growth modeling gets particularly challenging: newly deposited atoms might not find epitaxial sites directly at the surface, and further evolution could be needed before the reconstruction can be rebuilt. In general, a detailed knowledge of both kinetic and thermodynamic parameters is needed in order to understand the system evolution. For example, in the well-known $\operatorname{Si}(001)(2 \times 1)$ case, the monomer minimum-energy configuration does not correspond to an epitaxial site, ${ }^{6,7}$ and the nucleation of an additional layer passes through the creation of a suitable nucleus (see Ref. 8 and references therein). At low enough temperatures, however, the situation gets more complex since kinetics forces the creation of metastable diluted dimer structures. ${ }^{7,9-11}$

Reconstructions at semiconductor surfaces are not only critical in flat-film growth but also in determining threedimensional island stability. It has been shown that a complex, rebonded-step (RS) reconstruction takes place at $\mathrm{Ge}$ $\{105\}$ pyramid facets, ${ }^{12,13}$ and that such a reconstruction plays a fundamental role in lowering the energy of the threedimensional islands. The RS reconstruction strongly influences the morphology of the (105) surface, in eliminating the typical stepped profile of the as-cut geometry. As a result, the surface appears as rather flat, and it is characterized by a low surface energy, ${ }^{13,14}$ further decreased by compressive strain. ${ }^{15-17}$ Recent experiments ${ }^{18}$ showed that a very efficient step-flow growth mode must take place at the Ge-pyramid facets, since, before starting the transition to the dome shape, they always appear unstepped and very ordered in scanning tunneling microscopy (STM) images. A qualitative theoretical justification for the observed behavior came from $a b$ initio calculations, showing that fast adatom diffusion occurs at the Ge(105)RS surface. ${ }^{19}$ It is the aim of the present paper to go beyond the isolated adatom analysis, by investigating next-layer nucleation through the formation of larger structures, such as dimers and trimers, and by providing a direct connection between these two-dimensional (2D) clusters and the step-flow motion at such surface.

The paper is organized as follows. With the exception of Sec. II, dedicated to the methodology, the remainder of the work is focused on results' description and/or discussion. In particular, in Sec. III, we shall analyze possible ad-dimer configurations at the Ge(105)RS surface, while in Sec. IV, we shall explain and motivate our picture for next-layer nucleation, also considering a compressive strain applied at the surface, in an attempt to model the typical behavior expected at the facets of $\{105\}$ Ge pyramids on $\mathrm{Si}(001)$. Finally, before conclusions, in Sec. V, we discuss the connection between our nucleation model and the step-flow process at Gepyramid facets.

\section{COMPUTATIONAL DETAILS}

All the results presented in this work were obtained by $a b$ initio calculations performed using the VASP code, ${ }^{20-22}$ in which the Kohn and Sham equations are solved using a plane-wave basis, and ultrasoft pseudopotentials are utilized. $^{23,24}$ The exchange and correlation term was treated within Ceperley and Alder local-density approximation, as parametrized by Perdew and Zunger. ${ }^{25}$ The energy cutoff was set to $240 \mathrm{eV}$. The RS reconstructed (105) surface configuration was considered as a periodic arrangement of slabs, each composed of $14 \mathrm{ML}$, separated by $\sim 12 \AA$ of vacuum. The lattice parameter $a$ was set to the experimental value ( $a \sim 5.657 \AA$ ). Finally, dangling bonds at the lower surface were saturated with hydrogen atoms. In order to avoid lateral interactions, particularly important in the case where structures composed of two or more adatoms were considered, we 


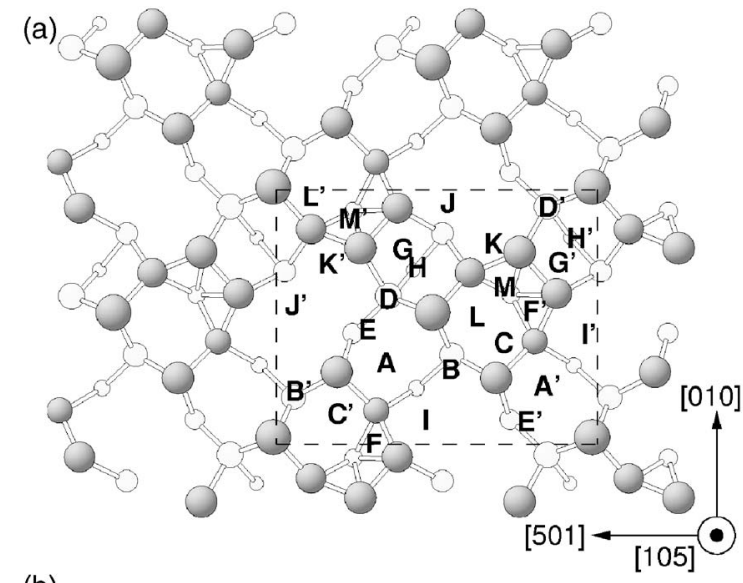

(b)

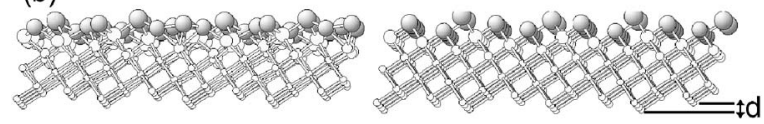

FIG. 1. a) Top view of the simulation cell. Substrate atoms are represented by white circles, and atoms of the U-shaped structures are painted in gray. Circles' radii are proportional to the distance to the observer. The A-M and $\mathrm{A}^{\prime}-\mathrm{M}^{\prime}$ letters correspond to the isolatedadatom possible positions, following the nomenclature proposed in Ref. 19. On the Ge(105)RS unit cell, there are 26 adsorption minima. The dashed lines encircle the unitary cell for the Ge(105)RS surface. (b) Lateral view of the (105) surface with the RS reconstruction (left panel) and in the as-cut configuration (right panel). With $d=0.555 \AA$, the distance between two adjacent layers is indicated.

used a large simulation cell, composed of $2 \times 2$ (105) unit cells, for a total of 296 atoms (plus the adatoms). The large lateral dimensions $(28.844 \times 22.627 \AA)$, see Fig. 1, made $\Gamma$-point results converged, as we directly verified. In geometry optimizations, all of the atoms were allowed to relax, but the four bottom layers were kept fixed.

Configurational optimizations were achieved by using a conjugate-gradient algorithm. Convergence at local minima or saddle points was declared when forces were less than $0.005 \mathrm{eV} / \AA$. In computing activation energies, we made use of the nudged elastic band (NEB) method. ${ }^{26}$ Accurate determination of the saddle-point energy was made possible by the NEB climbing-image refinement. ${ }^{27}$ Each configuration described as a minimum (saddle) was checked to display zero (one) negative curvatures by a standard normal-mode analysis.

\section{AD-DIMERS AT THE GE(105)RS SURFACE}

The (105)RS surface was already described in several publications (see, e.g., Refs. 12, 13, 15, and 16). Here, we only recall that a rebonding process reduces the number of dangling bonds of the as-cut geometry, giving raise to two, differently oriented, U-shaped structures (Uss) per surface unit cell, composed of three (001)-like rebonded dimers, which form the head and the two tails of the Uss. A top view of the surface is shown in Fig. 1, where the Uss are painted in gray to distinguish them from the substrate atoms. Such a reconstruction allows the surface to eliminate the stepped profile, inducing a rather flat morphology [see Fig. 1(b)].

As for the most popular $(001)(2 \times 1)$ surfaces, ${ }^{10}$ it is natural to consider dimers as possible initial seeds for further nucleation. For this reason, we first looked for several configurations for two adatoms on the Ge(105)RS, combining the already known single adatom minima proposed in Ref. 19. For each adsorption site (reported in Fig. 1 with labels A-M and $\mathrm{A}^{\prime}-\mathrm{M}^{\prime}$ ), we considered all the possible combinations involving some mutual perturbations. At a preliminary stage, in order to save computational time, we used a small simulation cell, halving the lateral dimensions of the one described in Sec. II (but using a $2 \times 3 \times 1 k$-point mesh, sufficient for reaching convergence).

Since, in the following, we shall often compare the energetics of structures characterized by a different number $N_{\text {ad }}$ of adatoms, we find it convenient to follow Yamasaki et al., ${ }^{7}$ associating with each configuration the energy per adatom $E$, defined by

$$
E=\frac{E_{\text {tot }}\left(N_{\mathrm{ad}}\right)-E_{\text {clean }}}{N_{\mathrm{ad}}},
$$

where $E_{\text {tot }}\left(N_{\mathrm{ad}}\right)$ is the total energy of a configuration with $N_{\text {ad }}$ adatoms added to the clean surface, and $E_{\text {clean }}$ is the cleansurface total energy. In Fig. 2(a), we report the energy per adatom of all the possible adatom pairs as computed for the smaller simulation cell, even if some of them cannot be described as ad-dimers, since no bonds between the adatoms are present. After selecting the more stable structures [the ones under the horizontal line in Fig. 2(a)], we reconverged them on the larger slab. The final energies with respect to the lowest configuration are shown in Fig. 2(b). Considering a larger cell is necessary to avoid lateral interactions which could alter the stability of each structure, as it is clearly demonstrated in Fig. 2(b) for the AL and AH arrangements. Further crucial evidence for this effect will be given in Sec. IV. The most stable two-adatom configuration is the so-called CL ad-dimer geometry which presents a (001)-dimer-like structure [see Fig. 3(a)]. We notice that the positions occupied by the two adatoms composing the ad-dimer closely correspond to the isolated-adatom local minima. ${ }^{19}$ The second more stable ad-dimer is AL, followed by AH and AI [see Figs. 3(b)-3(d), respectively]. They display an energy $\sim 0.1 \mathrm{eV}$ higher than CL. In particular, AL and AH are characterized by a quite close energy and this is due to the very similar geometry, differing only for the orientation of one adatom (in AL, it is oriented toward the center of the Uss, and in $\mathrm{AH}$ toward the outer region). In Fig. 2(a), a white diamond indicates the configuration obtained placing one adatom in $\mathrm{B}$ and one in $\mathrm{B}^{\prime}$ (the two stablest adatom sites ${ }^{19}$ ). It is clear that the reciprocal interactions of the two adatoms in $\mathrm{CL}, \mathrm{AL}, \mathrm{AH}$, and AI provide a strong stabilization, favoring the formation of dimers. The complex geometry of this reconstructed surface does not allow for a simple interpretation of the relative stability for the various configurations based on the degree of saturation only. Indeed, a good dangling-bond saturation could be associated with an unfavored overcoordination. This is the case of two adatoms po- 

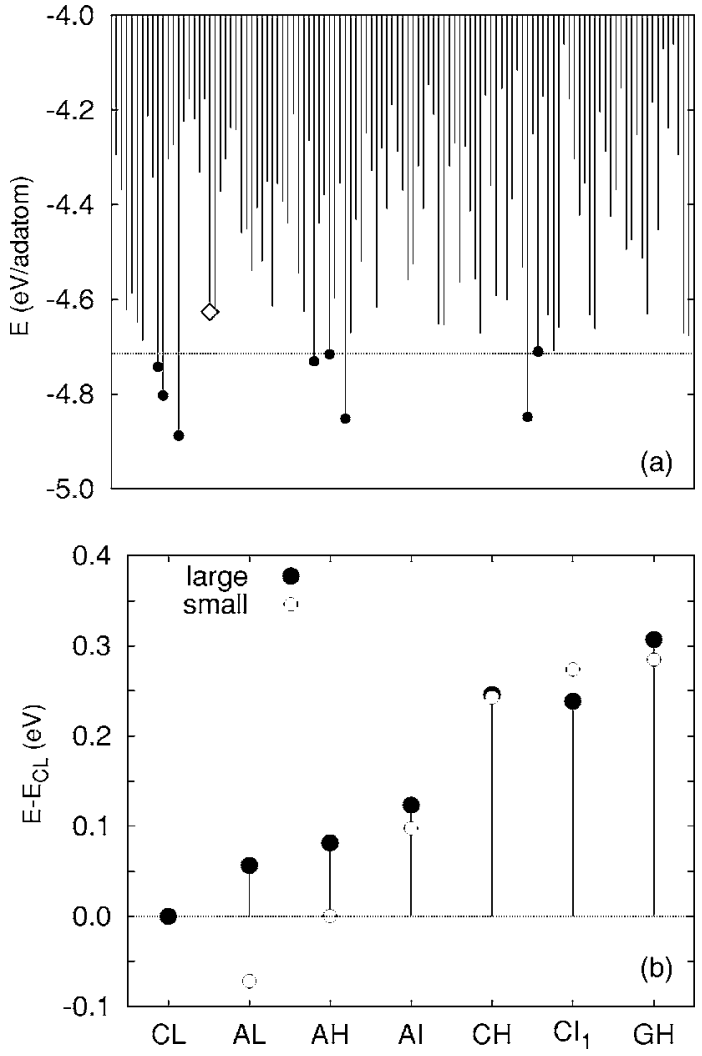

FIG. 2. (a) Binding energy per adatom for various two-adatom geometries on the $\mathrm{Ge}(105) \mathrm{RS}$ computed according to Eq. (1). The sampling has been performed on the smaller unit cell in order to search the most stable structures. The points indicated with the circles were also studied on the large simulation cell. (b) Total energy difference between various configurations and the lowestenergy one (CL dimer), for both the small and the larger simulation cell.

sitioned in two $\mathrm{B}$ sites (or in $\mathrm{B}$ and $\mathrm{B}^{\prime}$ ). They provide a better dangling-bond saturation with respect to $\mathrm{CL}, \mathrm{AL}$, and $\mathrm{AH}$, but at the same time, they induce pentacoordination of substrate atoms (the ones just under the adatoms). According to this consideration, a complex and not trivially detectable balance between gain and cost in the degree saturation and in the elastic energy makes CL the most stable minimum.

\section{NEXT-LAYER NUCLEATION AT THE GE(105)RS SURFACE AND AT GE-PYRAMID FACETS}

Once an ad-dimer is formed at the Ge(105)RS surface, we might ask whether larger 2D clusters can provide a further gain in energy. The geometry of the two most stable addimer sites (CL and AL in Fig. 3) immediately suggests a possible combined arrangement for an adtrimer, as illustrated in Fig. 4(c). In this structure, the adatom minimum called A (see Fig. 1) is now strongly delocalized, bridging the trench between two Uss's. The binding energy per atom is further decreased to $E \sim-4.95$ eV/adatom, $0.1 \mathrm{eV} /$ adatom lower than the most stable dimer CL [unstrained curve in Fig. 4(e)]. The adtrimer configuration looks like a promising stage toward the nucleation of the next (105)RS layer. Indeed, one
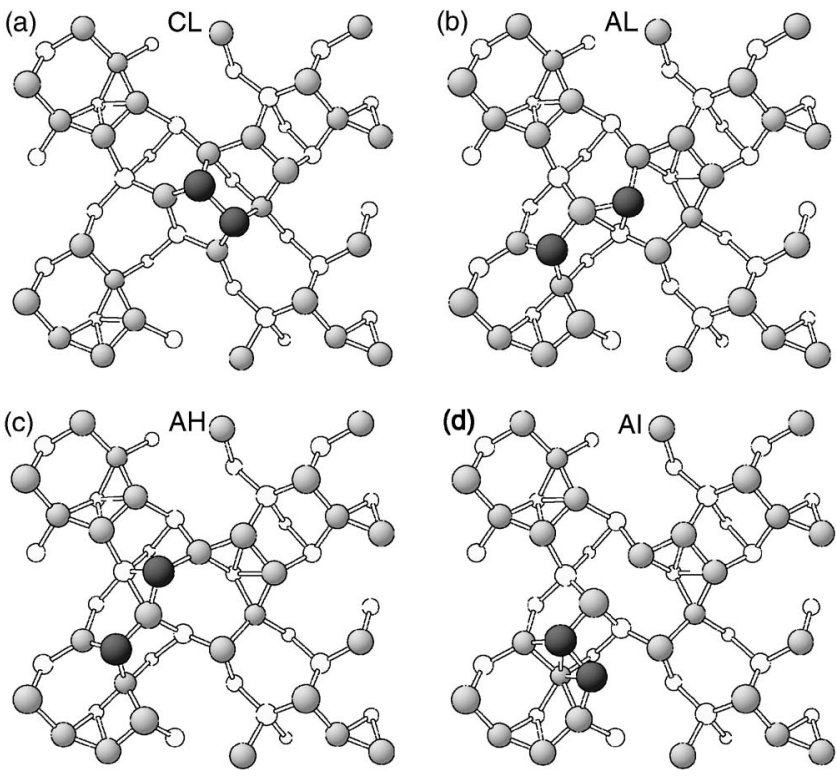

FIG. 3. Configurations obtained with $a b$ initio optimization for the four most stable structures, whose energy is reported in Fig. 2. The adatoms are painted in black to distinguish them from the substrate.

can easily recognize the typical footprints of a Uss (head + one of the two tails). Notice that a complete Uss could be formed without adding further adatoms if the dimer indicated by an arrow in Fig. 4(c) breaks, and one of its two atoms moves to complete the tail of the Uss. However, we verified such configuration to be highly unstable, due to an increase in the dangling bonds of the other atom composing the original dimer. Thus, another path must be followed. At first, we tried to add one and two further adatoms to the adtrimer configuration, but in this way we could not come up with a configuration with decreasing energy per adatom continuing the trend of Fig. 4(c) for $N_{\text {ad }}>3$. So, we considered the adtrimer as the real stable nucleus and we looked at ways for completing the new layer by using the $N_{\text {ad }}=3$ configuration as the key unit. An interesting result came out: the presence of a second, adjacent adtrimer located, as shown in Fig. 4(d), further reduces the energy per adatom, due to an effective lateral interaction produced by elastic effects. Due to the limited size of our simulation cell and to periodic boundary condition (PBC), we can actually draw this conclusion only for an infinite sequence of adtrimers, but the qualitative result is expected to be valid also for a finite number of adjacent adtrimers.

So far, we proposed a mechanism driving the evolution of the clean surface to an intermediate stable configuration [Fig. 4(d)], through a set of geometries obtained by simply placing an extra adatom on a suitable position, and by minimizing the energy. At each stage (one, two, three adatoms; set of aligned adtrimers), thermodynamics supported our guess, since the energy per adatom decreased with increasing $N_{\text {ad }}$ [see Fig. 2(e)]. Obviously, this scenario requires adatoms to easily reach the local minima positions involved. The recent theoretical evidence of fast and isotropic adatom diffusion on $\mathrm{Ge}(105) \mathrm{RS}$ at typical experimental temperatures ${ }^{19,28}$ $(T \gtrsim 700 \mathrm{~K})$ justifies our picture. However, we have not yet 

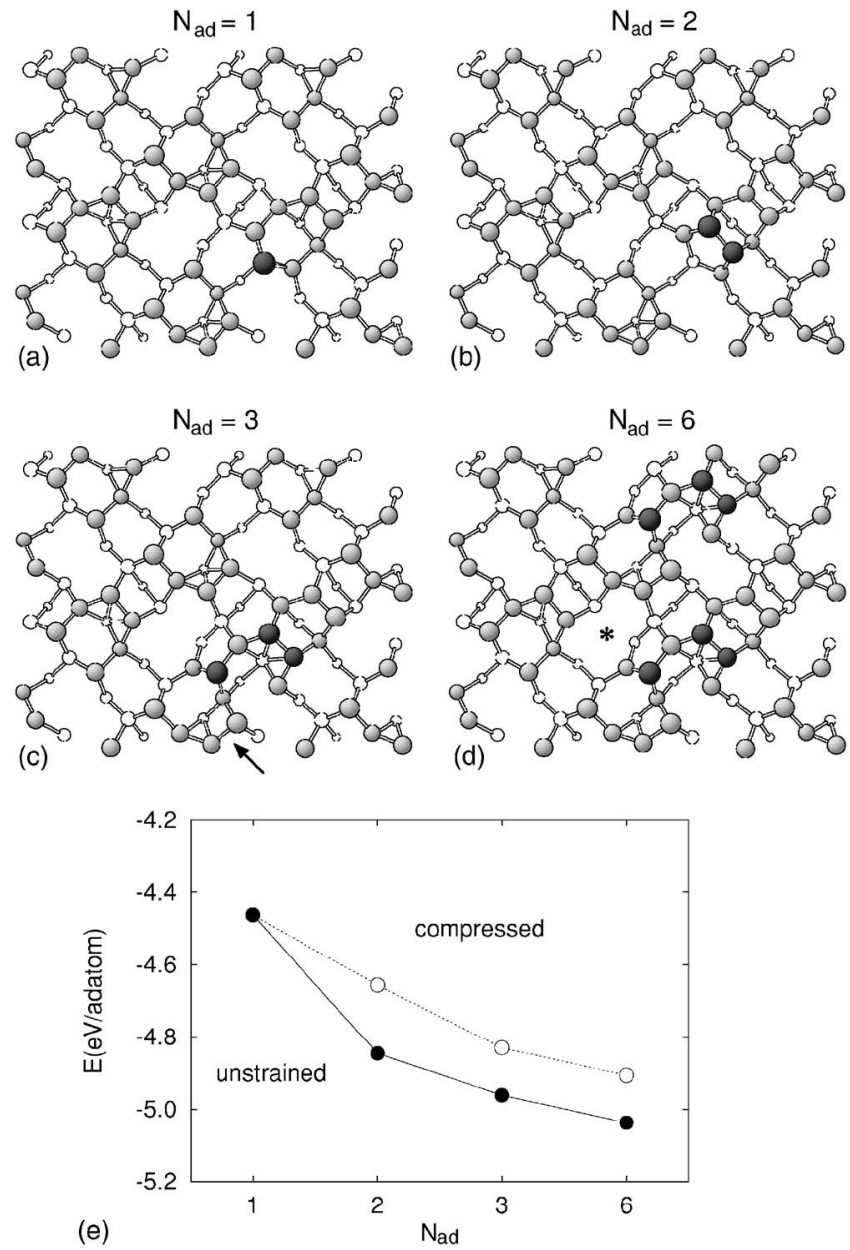

FIG. 4. Top view of the most stable structure for $N_{\mathrm{ad}}$ adatoms on the Ge(105)RS surface. (a) The most stable adatom configuration according to Ref. 19. (b) CL ad-dimer. (c) The adtrimer obtained combining CL and AL [see Fig. 3(d)]. (d) Two adjacent adtrimers; the asterisk $(*)$ indicates the position for another adatom which allows the further evolution of the growing surface. (e) Energy per adatom computed according to Eq. (1) for the structures reported in (a)-(d) panels.

shown how a full layer, displaying the perfect (105)RS geometry can be actually completed. In particular, we have only pointed out how adatoms are likely to group in set of adtrimers, which tend to be aligned along the [010] direction. Here, we propose a possible kinetic process able to trigger nucleation of the next layer. The mechanism needs adding a further adatom to the configuration of Fig. 4(d) and fast thermal surmounting of a kinetic barrier. The whole process is analyzed in Figs. 5(a)-5(d), the energetic along the path being computed by using the NEB method and shown in Fig. 5(e). Separated NEB calculations were run between each pair of adjacent local minima along the path. The starting configuration, representing the adjacent adtrimers previously described in Fig. 4(d) with an additional adatom being placed at the (*) position (isolated-adatom minimum $J$ ), is shown in Fig. 5(a). As a first step, the encircled dimer of Fig. 5(a) breaks, and one of its two atoms moves in the position indicated by an arrow to form a perfect Uss, as in Fig. 5(b). Such configuration is an extremely shallow minimum which gets

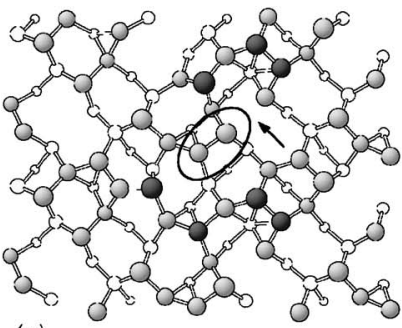

(a)

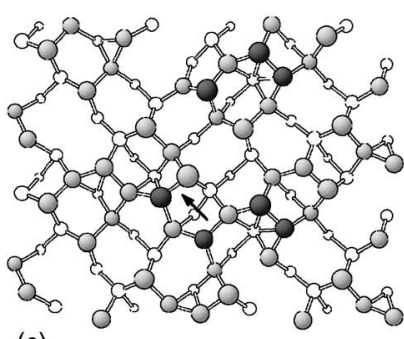

(c)

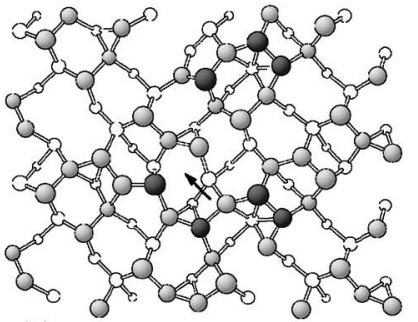

(b)

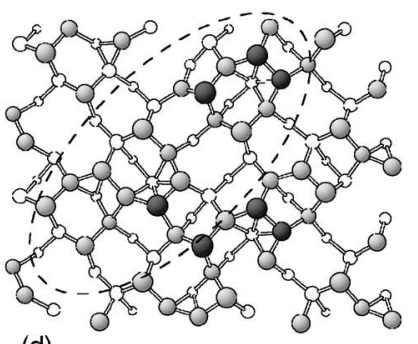

(d)

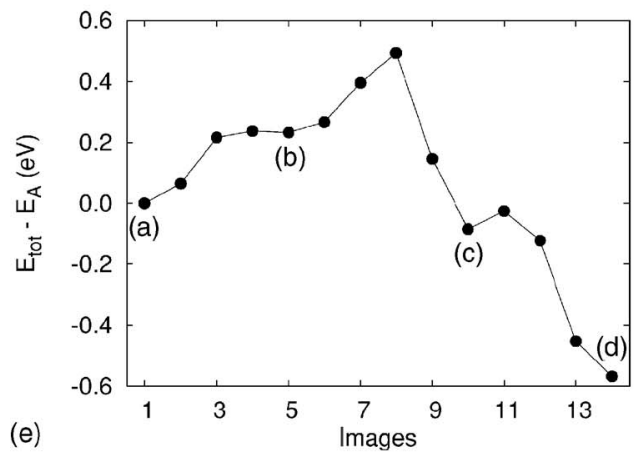

FIG. 5. Snapshots of the kinetic path leading to a Ge(105)RS patch one layer higher with respect to the substrate. Panels (a)-(d) represent local minima. The configuration in panel (a) [equivalent to Fig. 4(d) plus an adatom] displays the starting structure. The final geometry in panel (d) is characterized by an upper Ge(105)RS island (encircled by the dashed line) in which two Uss's of the new higher layer are easily detectable. The energy profile is reported in panel (e).

stabilized when the other atom, which just lost its neighbor, moves in the position indicated by the arrow in Fig. 5(b) and binds to the extra adatom, forming a new dimer [arrow in Fig. 5(c)]. This process, which requires surmounting an $\sim 0.3 \mathrm{eV}$ barrier, lowers the total energy with respect to the initial configuration by almost $0.5 \mathrm{eV}$. A final slight rearrangement, mainly involving a change in tilting of the newly formed (001)-like dimer, leads to the final configuration of Fig. 5(d), after passing a barrier of only $\sim 0.1 \mathrm{eV}$. Overall, as it is clear from Fig. 5(e), an effective barrier of $\sim 0.5 \mathrm{eV}$ is present from the initial to the final configuration. Notice that the mechanism leads to the formation of two Uss's [encircled by a dashed line in Fig. 5(d)]. The distance along the [105] direction between the upper atom of this new region with respect to the upper atom of the previous perfect substrate is increased by $\sim 0.576 \AA$, closely corresponding to the ideal distance between [105] planes in the bulk $(d \sim 0.555 \AA$ at our lattice parameter, see Fig. 1): we have added a twodimensional island while keeping the exact reconstruction by surmounting a barrier of $\sim 0.5 \mathrm{eV}$, which is lower than the 
typical values reported for isolated adatom diffusion [ $\geq 0.6 \mathrm{eV}$ (Refs. 19 and 28)]. This means that, on the typical time scale characterizing adatom diffusion, once two or more adjacent adtrimers and a further adatom are arranged in the initial configuration of Fig. 5(a), the evolution to the (105)RS geometry is extremely fast. By taking into account $\mathrm{PBC}$, the geometry shown in Fig. 5(d) is composed by an infinite set of adtrimers plus our newly formed 2D island, composed by exactly two Uss, and thus characterized by the typical dimensions of a (105)RS unit cell.

Recently, the combination of high-resolution STM images with extensive theoretical modeling led to a unified description of the growth mode for Ge pyramids on $\mathrm{Si}(001)$. Following Ref. 18 (but similar ideas are proposed also in Ref. 29 ), the nucleation of a new layer at the $\{105\}$ facets of $\mathrm{Ge}$ pyramids grown on $\mathrm{Si}(001)$ preferentially starts at the upper regions of the island. While simple thermodynamic arguments, based on the base-to-top lattice-parameter expansion within the Ge island, surely support the idea of an upper region energetically favored, and thus driving more material accumulation, a direct demonstration that nucleation is more likely to occur where the lattice parameter is closer to $\mathrm{Ge}$ bulk (i.e., at the top of island) was never reported. The analysis of isolated-adatom energetics ${ }^{19}$ is not sufficient: some local minima turn out to be more stable under compressive strain [a situation encountered also on the (001) surface ${ }^{30}$ ]. As shown below, the mechanism proposed here for nextlayer formation allows for a direct atomic-scale justification of the observed preference for nucleation toward the island top. In fact, we reconverged the configurations displayed in Fig. 4 , imposing an $\sim 4 \%$ in-plane compression, in an attempt to mimic the situation encountered at the Ge-pyramid base. The energetics of the system when one, two, or three adatoms are added to the clean surface is shown by a dotted line in Fig. 4(e). Once again, we observe that the gain in energy per atom is monotonic. However, more interestingly, it clearly appears that while for a single adatom (B site), there is basically no difference, for ad-dimers and adtrimers, the binding energies are all higher for the unstrained case with an energy gain of $\sim 0.2 \mathrm{eV} /$ adatom for the first and $\sim 0.15 \mathrm{eV} /$ adatom for the latter. Considering the $\{105\}$ facets of a $\mathrm{Ge}$ pyramid on $\mathrm{Si}(001)$, where adatoms can fast diffuse across both the compressed and the expanded region, ${ }^{19}$ it is, thus, clear that next-layer nucleation will be thermodynamically favored close to the island top, as shown by the experiments: ${ }^{18}$ adtrimers will tend to pack into the upper region, eventually (also helped by the geometry constrained given by the finite dimensions of the island) triggering the formation of one-layer higher 2D clusters at the facet.

Finally, we would like to stress that if in Ref. 13 a purely thermodynamic theoretical justification was given for the STM observation of a rather perfect (105)RS at the facets of Ge pyramids, here, we have shown that there are no kinetic limitations ${ }^{31}$ for the formation of the RS reconstruction, which is thus easily achievable even during growth.

\section{STEP FLOW AT GE PYRAMIDS FACETS}

Up to now, we modeled a possible kinetic mechanism driving the formation of a $2 \mathrm{D}$ cluster, involving a perfect
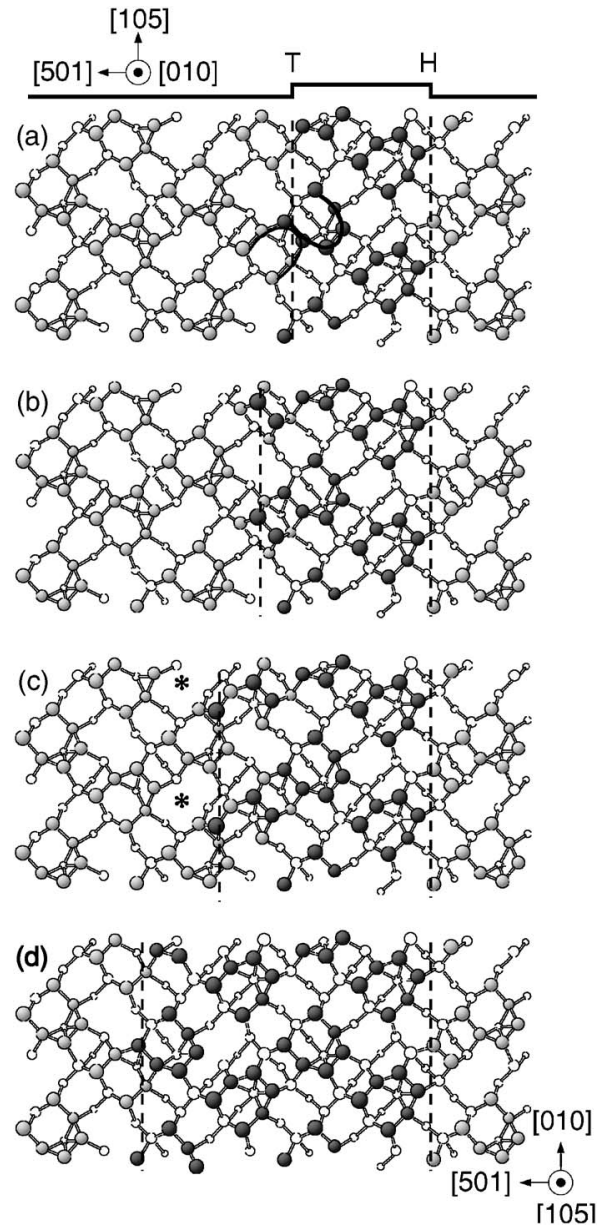

FIG. 6. Top view of Ge(105)RS. Atoms of the upper panel are in black, and atoms of the substrate in gray. (a) Perfect stripe of an upper Ge(105)RS layer. The two step edges are indicated by dashed lines: on the left there is a $T$ step, on the right an $H$ one. The boundaries of the two Uss crossing at the left edge, characterizing the lowest $T$ step, are traced with a black line. (b) Two ad-dimers are added on the lower Uss at the left edge. (c) After new adatom arrival, two adtrimers are present at the edge. (d) The $T$-step is grown along the [501] direction: a larger, perfectly reconstructed $\mathrm{Ge}(105) \mathrm{RS}$ terrace is now present in the middle of the slab.

Ge(105)RS patch and an adjacent adtrimer [see Fig. 5(d)]. Arrival of further adatoms in the close proximity of other adtrimers can trigger the lateral growth of this island. In particular, if such a mechanism takes place for any of the adtrimers, our island transforms into an actual stripe, as represented in Fig. 6(a). In order to emphasize that the stripe is an actual patch (infinite in [010] dimension) of a higher (105) layer, atoms in Fig. 6 are repainted by using dark filled circles for the upper middle terrace. The geometry of Fig. 6(a) is extremely interesting. In Ref. 32, indeed, the structure and energetics of steps on Ge(105)RS were investigated, attention being focused on the step orientation characterizing growth at the facets of $\{105\} \mathrm{Ge}$ pyramids. Depending on the edge position with respect to the underlying substrate, different step geometries can be obtained; for example, with step edges along [010] directions, two different types, called $T$ and $H$ in Ref. 32, are possible. For $T$ steps, the tails of the 
Uss in the upper terrace point toward the edge, while for $H$ steps, it is the head of the Uss which faces the edge. In studying the evolution of the $\{105\}$ pyramids facets, the major role is played by $T$ steps. ${ }^{32}$ Observing carefully the configuration reported in Fig. 6(a), the stripe formed according to our model is bounded on its left side precisely by the stablest $T$ step indicated by the classical-potential calculations of Ref. 32. Such step is easily recognizable, due to the characteristic crossing of two Uss, highlighted by continuous black lines in Fig. 6(a). This makes our nucleation model of direct relevance also for describing $T$-step propagation. Moreover, considering that, as already pointed out in the previous section, the nucleation of a new layer at the $\{105\}$ facets of $\mathrm{Ge}$ pyramids grown on $\mathrm{Si}(001)$ preferentially starts at the upper regions of the island, the facets being then completed by a fast step-flow mechanism of $T$ steps, such a process is directly connected to the evolution of $\{105\}$ pyramid facets. Indeed, if adatoms wandering along the terrace delimited by the $T$ step form a dimer (and then a trimer) in the position indicated in Fig. 6(b) [and then Fig. 6(c)], i.e., directly above the lowest of the two crossing Uss's, the same sequence for creation of the next (105)RS layer described in Fig. 5 can take place exactly at the $T$-step edge, with the adjacent adtrimers now naturally aligned by the presence of the step. After the arrival of a further adatom per adtrimer in the position indicated with (*) in Fig. 6(d) a kinetic mechanism analogous to the one analyzed in Fig. 5 leads to perfect growth of the $T$ step in the [501] direction, and to a consequent enlargement of the (105)RS higher terrace. By assuming the barrier for such process to be of the same order $(\sim 0.5 \mathrm{eV})$ of the one computed for the slightly simpler geometry of Fig. 5, we can conclude that in the presence of $T$ steps at the (105)RS surface, the ordered growth of the steps is fast, being simply limited by the flux of arriving adatoms. The fact that pyramid facets are never observed to be uncompleted in STM images, ${ }^{19}$ if not close to the transition to the dome morphology, is here suggested to be caused by the quick $T$-step flow from the top to bottom of the Ge island, yielding facet completion.

\section{CONCLUSIONS}

In this paper, we have proposed an atomic-scale path leading to next-layer nucleation on Ge(105)RS surfaces. A suitable adtrimer configuration was individuated, and the tendency toward forming clusters of aligned adtrimers was demonstrated. A low-barrier kinetic mechanism leading to the formation of actual 2D islands displaying the (105)RS reconstruction was proposed and shown to be faster than typical adatom diffusion. The process was then linked to step flow, discussing its implications on the growth kinetics of $\mathrm{Ge}(105)$ pyramids on $\mathrm{Si}(001)$.

\section{ACKNOWLEDGMENTS}

We would like to thank Leo Miglio for help in result interpretation and for a careful reading of this paper, G. Henkelman for his useful scripts used in the normal-mode analysis, and D. B. Migas for some preliminary work. Computational support from CINECA (INFM project "Iniziativa Calcolo Parallelo" 2004 and 2005) and CILEA (under the L-NESS account) are gratefully acknowledged, together with financial support from the Cariplo Foundation.
*Electronic address: francesco.montalenti@unimib.it

${ }^{1}$ T. Michely and J. Krug, Islands, Mounds and Atoms (Springer, Berlin, 2004).

${ }^{2}$ T. Ala-Nissila, R. Ferrando, and S. C. Ying, Adv. Phys. 51, 949 (2002).

${ }^{3}$ A. F. Voter, Phys. Rev. B 34, 6819 (1986).

${ }^{4}$ F. Montalenti, Surf. Sci. 543, 141 (2003).

${ }^{5}$ G. P. Srivastava, Rep. Prog. Phys. 60, 561 (1997).

${ }^{6}$ G. Brocks, P. J. Kelly, and R. Car, Phys. Rev. Lett. 66, 1729 (1991).

${ }^{7}$ T. Yamasaki, T. Uda, and K. Terakura, Phys. Rev. Lett. 76, 2949 (1996).

${ }^{8}$ Raj Ganesh S. Pala and F. Liu, Phys. Rev. Lett. 95, 136106 (2005).

${ }^{9}$ P. J. Bedrossian, Phys. Rev. Lett. 74, 3648 (1995).

${ }^{10}$ G. Brocks and P. J. Kelly, Phys. Rev. Lett. 76, 2362 (1996).

${ }^{11}$ A. P. Smith and H. Jónsson, Phys. Rev. Lett. 77, 1326 (1996).

${ }^{12}$ Y. Fujikawa, K. Akiyama, T. Nagao, T. Sakurai, M. G. Lagally, T. Hashimoto, Y. Morikawa, and K. Terakura, Phys. Rev. Lett. 88, 176101 (2002).

${ }^{13}$ P. Raiteri, D. B. Migas, L. Miglio, A. Rastelli, and H. von Känel, Phys. Rev. Lett. 88, 256103 (2002).

${ }^{14}$ T. Hashimoto, Y. Morikawa, Y. Fujikawa, T. Sakurai, M. G. Lagally, and K. Terakura, Surf. Sci. 513, L445 (2002).
${ }^{15}$ D. B. Migas, S. Cereda, F. Montalenti, and L. Miglio, Surf. Sci. 556, 121 (2004).

${ }^{16}$ C. V. Ciobanu, V. B. Shenoy, C. Z. Wang, and K. M. Ho, Surf. Sci. 544, L715 (2003).

${ }^{17}$ O. E. Shklyaev, M. J. Beck, M. Asta, M. J. Miksis, and P. W. Voorhees, Phys. Rev. Lett. 94, 176102 (2005).

${ }^{18}$ F. Montalenti, P. Raiteri, D. B. Migas, H. von Känel, A. Rastelli, G. Costantini, C. Manzano, U. Denker, O. G. Schmidt, K. Kern et al., Phys. Rev. Lett. 93, 216102 (2004).

${ }^{19}$ F. Montalenti, D. B. Migas, F. Gamba, and L. Miglio, Phys. Rev. B 70, 245315 (2004).

${ }^{20}$ G. Kresse and J. Hafner, Phys. Rev. B 47, 558 (1993).

${ }^{21}$ G. Kresse and J. Hafner, Phys. Rev. B 49, 14251 (1994).

${ }^{22}$ G. Kresse and J. Furthmüller, Phys. Rev. B 54, 11169 (1996).

${ }^{23}$ D. Vanderbilt, Phys. Rev. B 41, 7892 (1990).

${ }^{24}$ G. Kresse and J. Hafner, J. Phys.: Condens. Matter 6, 8245 (1994).

${ }^{25}$ J. P. Perdew and A. Zunger, Phys. Rev. B 23, 5048 (1981).

${ }^{26}$ H. Jónsson, G. Mills, and K. W. Jacobsen, in Classical and Quantum Dynamics in Condensed Phase Simulations, edited by B. J. Berne, G. Ciccotti, and D. F. Coker (World Scientific, Singapore, 1998), p. 383.

${ }^{27}$ G. Henkelman, B. P. Uberuaga, and H. Jónsson, J. Chem. Phys. 113, 9901 (2000). 
${ }^{28}$ G. H. Lu, M. Cuma, and F. Liu, Phys. Rev. B 72, 125415 (2005).

${ }^{29}$ V. Zela, I. Pietzonka, T. Sass, C. Thelander, S. Jeppesen, and W. Seifert, Physica E (Amsterdam) 13, 1013 (2002).

${ }^{30}$ A. van de Walle, M. Asta, and P. W. Voorhees, Phys. Rev. B 67, 041308(R) (2003).

${ }^{31}$ We did not repeat the calculation for the kinetic path of Fig. 5 applying a biaxial compressive stress at the surface. Since ac- cording to Ref. 19 the kinetic barriers for adatoms diffusion on Ge(105)RS presented no significant difference between the unstrained and strained case, we drew this conclusion also for the mechanism reported in this work.

${ }^{32}$ S. Cereda, F. Montalenti, and L. Miglio, Surf. Sci. 591, 23 (2005). 